\title{
Active network management: planning under uncertainty for exploiting load modulation
}

\author{
Quentin Gemine, Efthymios Karangelos, Damien Ernst, Bertrand Cornélusse \\ Department of Electrical Engineering and Computer Science, \\ University of Liège, Liège 4000, Belgium \\ \{qgemine, e.karangelos, dernst, bertrand.cornelusse\}@ulg.ac.be
}

\begin{abstract}
This paper addresses the problem faced by a distribution system operator (DSO) when planning the operation of a network in the short-term. The problem is formulated in the context of high penetration of renewable energy sources (RES) and distributed generation (DG), and when flexible demand is available. The problem is expressed as a sequential decisionmaking problem under uncertainty, where, in the first stage, the DSO has to decide whether or not to reserve the availability of flexible demand, and, in the subsequent stages, can curtail the generation and modulate the available flexible loads. We analyze the relevance of this formulation on a small test system, discuss the assumptions made, compare our approach to related work, and indicate further research directions.
\end{abstract}

\section{Introduction}

In Europe, concern about the environmental impact of the electricity industry is currently driving the growth of renewable electricity generation through a number of financial support mechanisms [1]. Such incentives have resulted in the on-going installation of wind and solar power generation resources at the distribution level of the electricity network. This development calls for evolution of distribution network planning and operational strategies, in order to accommodate the energy produced by such DG resources.

The dominant doctrine for the distribution network planning and operation has been the fit and forget approach [2]. Under this approach, adequate investments in network components (i.e., lines, cables, transformers, etc.) must be made in order to always avoid congestion and voltage problems. To that end, network planning is made with respect to a set of critical scenarios consisting of DG production and demand levels. In this manner, sufficient operational margins are always ensured [3]. Nevertheless, with the rapid growth of DG resources, the preservation of such conservative margins comes at continuously increasing network reinforcement costs.

In order to avoid potentially prohibitive network reinforcement costs, several active network management (ANM) strategies have recently been proposed as alternatives to the fit and forget approach. The principle of ANM is to address congestion and voltage issues via short-term decision-making policies [2], developed on the basis of the optimal power flow (OPF) problem formulation [4]. Liew and Strbac [5] considered a deterministic problem formulation with the objective of minimizing the market value of the curtailed DG energy production. Dolan et al. [6] developed a deterministic, OPFbased adaptation of the 'last-in, first-off' operating practice. A multi-period framework has been considered to account for the effects of the variability of renewable DG resources [7], [8], [9]. In this context, the problem of maximizing the utilization of the available DG inflow has been addressed [7], as well as the minimization of the energy losses in the electrical network [8]. The integration of advanced network constraints, including fault levels and N-1 security, has been established within this multi-period framework [9].

In these references, ANM schemes maintain the system within operational limits in a quasi real-time by relying on the curtailment of wind or solar generation. Curtailment of renewable energy may, however, be very controversial on an environmental point of view and should probably be considered as a last resort. In that mindset, it would be worth investigating ANM schemes that could also exploit the flexibility of the load, so as to decrease the reliance on generation curtailment. Let us consider a typical case where, due to a high production of renewable energy, the distribution network sends an amount of power to the transmission network that creates congestion at the MV/HV transformer. By asking flexible loads of the distribution network (DN) to increase their consumption at that time, the congestion could potentially be relieved without having to rely on renewable generation curtailment. Wellknown examples of flexible loads are electric heaters, boilers, and electric car batteries. It is worth noting that exploiting flexible loads within an ANM scheme comes with several challenges. One such challenge is that modulating a flexible load at one time is often going to influence its modulation range at subsequent times. This is because flexible loads are often constrained to consume a specific amount of energy over a certain duration. In the above example, this would imply that after increasing the consumption of the flexible loads during a certain time period, the DSO would be constrained to later decrease their consumption, which may significantly aggravate congestion. In this context, it is therefore important for a DSO not to take myopic decisions, but to make decisions by planning over a relevant time horizon. Due to 
the uncertainty of the future power injections from renewable sources relying on natural phenomena (wind, sun, etc.) and of the power consumption of the loads, this would necessarily imply for the DSO to implement ANM scheme able to plan adequately control actions under uncertainty, which may be very challenging.

In this paper, we consider that the DSO can rely on both generation curtailment and load modulation for the active management of the network. We focus on a specific setting where the DSO can procure the right to control the demand pattern of the flexible loads, in exchange for an availability fee. We also assume that the DSO has to pay a fee per MWh of energy curtailed, and that the fee depends on the market price of electricity at the time of curtailment. In this context, we discuss the way the DSO should plan the control actions to operate safely the network at minimal cost. In the following section, we describe the main characteristics of the ANM problem. Following this, we formalize the problem as an optimal sequential decision-making problem under uncertainty, and discuss resolution strategies. The paper concludes with simulation results obtained on a six bus test system. These results show that proper management of the uncertainty can be very beneficial.

\section{Problem description}

We focus on the problem faced by a DSO that aims at maintaining the network infrastructure within operational limits over a one day horizon. We consider the possibility that the DSO can impose power curtailment instructions on the DG resources, in exchange for financial compensation. From the alternative payment structures outlined in [10], we adopt a scheme where the per unit compensation is defined as the electricity market price for the curtailment period.

In addition, we account for the possibility for the DSO to procure the right to control the demand pattern of the flexible loads in exchange for an availability fee. Unlike non flexible loads, we consider that a load participating in this flexibility market must be able to precisely follow both the instructed modulations if the flexibility offer is selected by the DSO, or the baseline demand profile, if this option is not selected. There is therefore no uncertainty introduced by these loads. In summary, we characterize the flexible operating region of such loads through the following features:

- A baseline demand profile, to be followed with certainty unless instructed by the DSO.

- Upward and downward demand modification limits per period.

- A net energy balance requirement, so that any instruction by the DSO should not modify the net energy volume consumed by a flexible load over the optimization horizon, with respect to the baseline profile.

The time horizon covered in this problem is accounted by

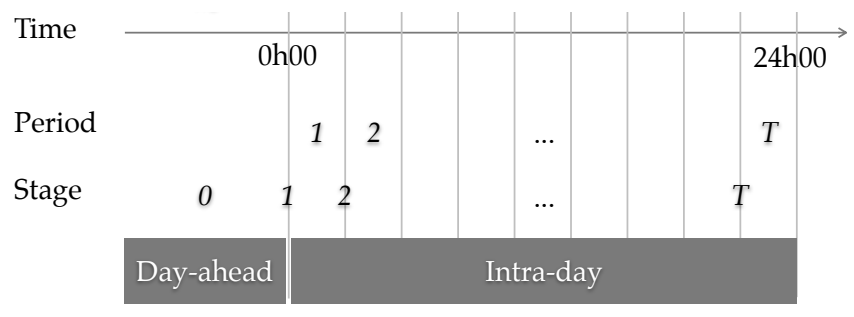

Fig. 1: Decision framework.

optimizing the operation of the system over a set of $t \in$ $[1, T]$ discrete periods. The decision framework illustrated in Figure 1 consists of:

- An ex-ante stage, in which the provision of load flexibility is settled, which can coincide with the termination of the usual day-ahead trading process.

- A series of recourse opportunities, at the start of every market period, during which the DSO can rely to submit curtailment orders.

Finally, we have a set of $k \in[0, T]$ decision stages. At the ex-ante decision stage $(k=0)$, the energy inflow from the DG units and the demand of non-flexible loads are uncertain for all the periods within the optimization horizon $t \in[1, T]$. Moreover, at each recourse stage $k \in[1, T]$, these quantities are assumed to be known with certainty for periods $t \in[1, k]$ but are uncertain for the subsequent time interval $t \in[k+1, T]$. Within each period, the behavior of the network is modeled by steady-state AC power flow equations, to allow us to state operational limits on voltage, current, and power.

\section{Sequential decision-making under uncertainty}

We gradually define the elements of an optimal sequential decision-making problem, and then enrich the problem by modeling the uncertainty explicitly, to allow us to state the detailed formulation of the targeted application in the next section.

\section{Optimal sequential decision-making}

We place ourselves in a setting where we want to control the evolution of a system over a time horizon discretized in $T$ time steps. Sequential decision-making problems arise when the decisions taken before the time $t<T$ influence the decisions available between times $t$ and $T$. Therefore, the problem cannot be decomposed into a sequence of $T$ independent problems.

Let $x \in \mathcal{X}$ be the state variable, that is the vector describing the system, and $u \in \mathcal{U}(x) \subset \mathcal{U}$ the vector describing a decision, or control action, which can be taken to modify the state $x$. By definition, the state contains enough information so that knowing the control action at time $t$ and the state at the previous period, respectively $u_{t}$ and $x_{t-1}$, it is always possible to compute $x_{t}, \forall t \in\{1,2, \ldots, T\}$. In other words, the evolution 


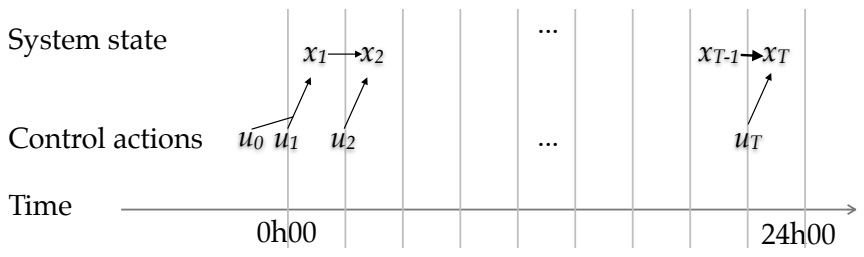

Fig. 2: States and control actions.

of the system is guided by the function

$$
f: \mathcal{X} \times \mathcal{U} \rightarrow \mathcal{X}
$$

such that

$$
x_{t}=f\left(x_{t-1}, u_{t}\right) .
$$

This is illustrated schematically in Figure 2. In our specific framework, given that the initial decision stage does not coincide with a specific period, we adapt the previous notation for $x_{1}$ and introduce $f_{1}: \mathcal{U}_{0} \times \mathcal{U} \rightarrow \mathcal{X}$, to obtain

$$
\begin{aligned}
x_{1} & =f_{1}\left(u_{0}, u_{1}\right) \\
x_{t} & =f\left(x_{t-1}, u_{t}\right), \forall t \in\{2, \ldots, T\}
\end{aligned}
$$

A sequence of control actions $\left(u_{0}, u_{1}, u_{2}, \ldots, u_{T}\right)$ is admissible if $x$ stays within $\mathcal{X}$ for $t \in\{1,2, \ldots, T\}$. To ease notations, we denote $\left(u_{0}, u_{1}, u_{2}, \ldots, u_{t}\right)$ by $\mathbf{u}_{[t]}$ and $\left(x_{1}, x_{2}, \ldots, x_{t}\right)$ by $\mathbf{x}_{[t]}$ and pose $\mathcal{T}=\{2,3, \ldots, T\}, \mathbf{x}=\mathbf{x}_{[T]}, \mathbf{u}=\mathbf{u}_{[T]}$. To guide the choice of a sequence of control actions from all the admissible sequences, we need to define an objective function

$$
J: \mathcal{X}^{T} \times \mathcal{U}_{0} \times \mathcal{U}^{T} \rightarrow \mathbb{R} .
$$

The optimal sequential decision-making problem can then be summarized as

$$
\begin{array}{ll}
\min _{\mathbf{x}, \mathbf{u}} & J(\mathbf{x}, \mathbf{u}) \\
\text { s.t. } & x_{1}=f\left(u_{0}, u_{1}\right) \\
& x_{t}=f_{1}\left(x_{t-1}, u_{t}\right), \quad \forall t \in \mathcal{T} \\
& x_{t} \in \mathcal{X}_{t}, \quad \forall t \in\{1\} \cup \mathcal{T} \\
& u_{t} \in \mathcal{U}_{t}\left(x_{t-1}\right), \quad \forall t \in \mathcal{T} \\
& u_{1} \in \mathcal{U}_{t}\left(u_{0}\right) \\
& u_{0} \in \mathcal{U}_{0}
\end{array}
$$

In this work, we consider that $u$ contains generation curtailment and flexible load modulation decisions and that $x$ contains the flexibility availability indicator and the energy already supplied to each flexible load. One exception is that $u_{0}$ only contains flexibility provision decisions. All the variables describing the electrical state of the system are also included in $x$. As we consider steady state operation of the electrical system, these variables are time independent knowing the energy already supplied to each flexible load. Hence, the transition function $f$ propagates the flexibility availability indicator and accumulates the energy supplied to each flexible load, whereas $x \in \mathcal{X}$ ensures that the power flow equations and operational limits are satisfied. Finally, $J(\mathbf{x}, \mathbf{u})$ is the sum of curtailment and flexibility provision costs, and is decomposable by period.

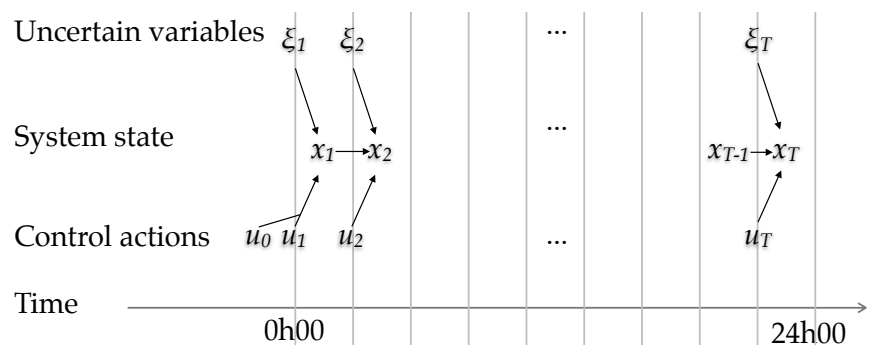

Fig. 3: States, control actions and uncertainty.

\section{Handling uncertainty}

Many problems can be formulated as computing an optimal sequence of decisions over a time horizon tarnished with uncertainty, and allowing a series of recourse opportunities to adapt the sequence of decisions to the outcome of the random process representing the uncertainty. One of the first power system applications where uncertainty was explicitly considered was the unit commitment problem [11]. In the mathematical programming community, this type of problem is referred to as a multi-stage stochastic program (MSP). A classical way to formulate a MSP is to optimize the expectation of an objective function of several variables over an uncertainty set of the parameters, the variables being required to satisfy constraints for all possible realizations of the uncertain parameters, and to impose the structure of the recourse opportunities. In doing so, control actions are taken so as to hedge against the possible evolutions of the exogenous variables of the system.

Let $\xi$ be a stochastic process defined on the probability space $(\boldsymbol{\Omega}, \mathbf{F}, \mathbf{P}) . \xi_{t}$ is a realization of $\xi$ at time $t \in\{1,2, \ldots, T\}$ and the notation $\xi_{[t]}$ represents the sequence $\left(\xi_{1}, \ldots, \xi_{t}\right)$. We now consider that the evolution of the system as well as the control actions are functions of those exogenous variables. This is expressed by $x_{t}\left(\xi_{[t]}\right)$ and $u_{t}\left(\xi_{[t]}\right)$, and is illustrated schematically in Figure 3. The optimal sequential decisionmaking problem (1)-(7) can be adapted to handle uncertainty as follows:

$$
\begin{aligned}
\min _{\mathbf{x}\left(\xi_{[T]}\right)} & \mathbb{E}_{\xi_{[T]}}\left\{J\left(\mathbf{x}\left(\xi_{[T]}\right), \mathbf{u}\left(\xi_{[T]}\right), \xi_{[T]}\right)\right\} \\
\mathbf{u}\left(\xi_{[T]}\right) & \\
\text { s.t. } & x_{1}\left(\xi_{1}\right)=f_{1}\left(u_{0}, u_{1}\left(\xi_{1}\right), \xi_{1}\right) \\
& u_{0} \in \mathcal{U}_{0} \\
& u_{1}\left(\xi_{1}\right) \in \mathcal{U}_{t}\left(u_{0}, \xi_{1}\right) \\
& \forall t \in \mathcal{T}, \\
& x_{t}\left(\xi_{[t]}\right)=f\left(x_{t-1}\left(\xi_{[t-1]}\right), u_{t}\left(\xi_{[t]}\right), \xi_{t}\right) \\
& u_{t}\left(\xi_{[t]}\right) \in \mathcal{U}_{t}\left(x_{t-1}\left(\xi_{[t-1]}\right)\right) \\
& \forall t \in\{1\} \cup \mathcal{T} \\
& x_{t}\left(\xi_{[t]}\right) \in \mathcal{X}_{t}
\end{aligned}
$$

As the process $\xi_{[T]}$ does not have a finite number of realizations in the applications that we target, this formulation leads to an infinite dimensional optimization problem [12]. We now present a common method of approximating this problem. 


\section{Scenario tree based approximation}

We approximate the random process with a discrete distribution and a finite support in order to build scenarios (Figure $4 \mathrm{a}$ ) of possible realizations of the random process, and to optimize the objective function only for the realizations of the random process represented by these scenarios. Nonanticipativity constraints are imposed to enforce the structure of the recourse opportunities: for a given recourse opportunity, all decisions related to scenarios with a common past must be equal. Each recourse opportunity is called a stage. To facilitate the representation of these constraints, the scenarios are often clustered in a scenario tree as shown in Figure 4b. The depth

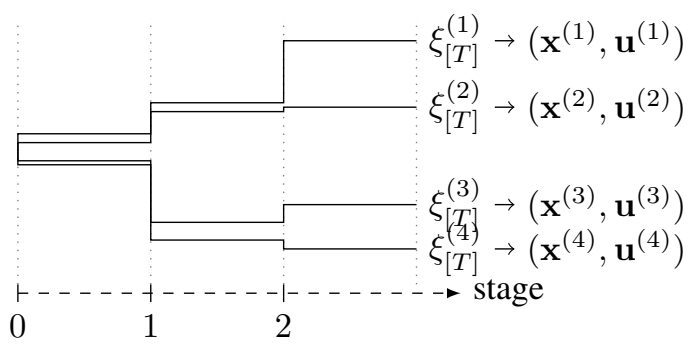

(a) Representation of different possible sequences of realizations thanks to scenarios. A sequence of decisions corresponds to each scenario.

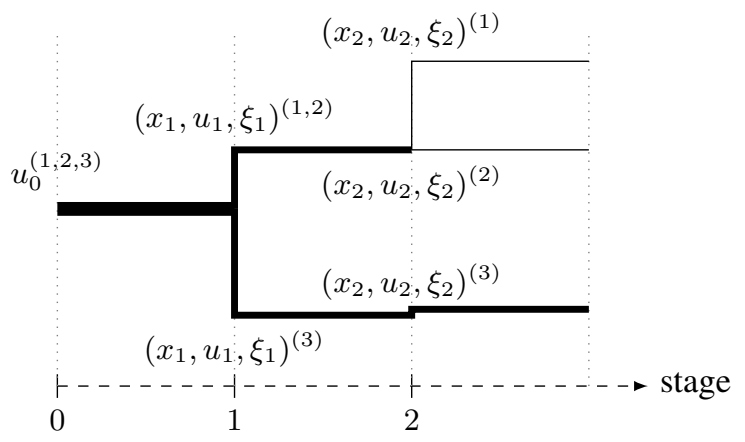

(b) Hierarchical clustering into a scenario tree. A unique decision corresponds to each node of the tree.

Fig. 4: Scenarios and stages

of the tree constructed by this approach is equal to $T$. With the exception of the root node, which corresponds to the dayahead, each node of the tree corresponds to a realization of the random process at a given stage and has at least one son for $t=1, \ldots, T-1$. A scenario contains successive realizations of the random variable, and is represented in the tree by going from the root node and taking one son at each subsequent level down to the final level. Scenario $k$ is denoted by $\xi_{[T]}^{(k)}$ with $k \in\{1,2, \ldots, K\}$ where $K$ is the number of leaves in the tree. Each node $n \in\{1,2, \ldots, N\}$, where $N$ is the total number of nodes in the tree truncated at the root node, has an associated occurrence probability $w_{n}$ and stage $t$. The node $n=0$ corresponds to the root node. We use subscript $n$ to reference state, control actions and exogenous variables at node $n$ of the tree, and use subscript $[n]$ to refer to the sequence of states, control actions and exogenous variables from the root of the scenario tree to node $n$. Using this notation, the scenario tree based approximation of problem (8)-(14) is

$$
\begin{array}{ll}
\min _{\mathbf{x}, \mathbf{u}} & \sum_{k=1}^{K} w_{k} J\left(\mathbf{x}^{(k)}, \mathbf{u}^{(k)}, \xi_{[T]}^{(k)}\right) \\
\text { s.t. } & x_{n}=f\left(u_{0}, u_{n}\right), \forall n \in \mathcal{C}(0) \\
& u_{0} \in \mathcal{U}_{0} \\
& x_{n^{\prime}}=f\left(x_{n}, u_{n^{\prime}}, \xi_{\left[n^{\prime}\right]}\right) \forall n^{\prime} \in \mathcal{C}(n), \forall n \in \mathcal{N} \\
& x_{n} \in \mathcal{X}_{n}, \forall n \in \mathcal{N} \\
& u_{n^{\prime}} \in \mathcal{U}_{n^{\prime}}\left(x_{n}\right), \forall n^{\prime} \in \mathcal{C}(n), \forall n \in \mathcal{N}
\end{array}
$$

where $w_{k}$ is the probability of the terminal node in scenario $k$, $\mathcal{C}(n)$ is the set of successors of node $n$, and $\mathcal{N}=\{1,2, \ldots, N\}$ is the set of nodes of the scenario tree truncated at its root node.

\section{Detailed optimization model}

This section details the components of the compact formulation presented in problem (15)-(20).

\section{Network topology}

The network is represented by a graph, where the set of vertices $\mathcal{B}$ represents the electrical buses and the set of edges $\mathcal{L}$ contains the links connecting buses. A nominal voltage level is associated with each bus. In this work we focus on the MV level network. Several devices can be connected to each bus. We distinguish three types of devices: generators, static loads, and flexible loads. These devices represent either a single physical element, such as a wind turbine, or the aggregation of elements of the same type connected to an individual bus, such as residential loads at the low voltage (LV) level. $\mathcal{G}, \mathcal{S}$ and $\mathcal{F}$ represent the set of generators, static loads, and flexible loads, respectively. $\mathcal{G}(b), \mathcal{S}(b)$ and $\mathcal{F}(b)$ represent the devices of each type connected to bus $b$, respectively. Links are transformers, lines or cables. No special notation is required to distinguish different types of links, as these are directly translated into the values of the admittance matrix.

\section{Parameters}

Let $P_{g, n} \geq 0$ be the injection of generator $g$ and $P_{s, n} \geq$ 0 and $P_{f, n} \geq 0$ the off-takes of the static and flexible loads, respectively. Off-takes of each flexible load are known with certainty and are characterized by the time-dependent minimum, $\underline{P}_{f, t}$, and maximum, $\bar{P}_{f, t}$, power levels ${ }^{1}$. On the other hand, wind and solar power injections and off-takes of static loads are considered uncertain. In addition, we consider that all devices operate at constant power factor $\cos \phi_{d}$ and their reactive power injections are thus defined by:

$$
Q_{d, n}=\tan \phi_{d} P_{d, n}, \forall d \in \mathcal{G} \cup \mathcal{S} \cup \mathcal{F} .
$$

All electrical parameters are gathered in the admittance ma-

\footnotetext{
${ }^{1}$ For simplicity, flexibility bids cover the whole time horizon. Removing this restriction does not change the nature of the optimization problem.
} 
$\operatorname{trix}^{2} Y \in \mathbb{C}^{|\mathcal{B}| \times|\mathcal{B}|}$. Operational limits are $\underline{V}_{b}$ and $\bar{V}_{b}$, the minimum and maximum voltage magnitude, respectively, and $\bar{I}_{l}$, the maximum current.

The cost of flexibility availability and curtailment are $C_{f}^{f l e x}$, and $C_{g, t}^{c u r t}$, respectively. At any decision stage $k \in[1, T]$, the cost would be uncertain, given that the curtailed energy would have to be compensated at the real-time market price.

\section{State variables}

The state variable is

$$
x=\left(\mathbf{e}^{\top}, \mathbf{y}^{\top}, \mathbf{p}^{\top}, \mathbf{q}^{\top}, \mathbf{V}^{\top}\right)
$$

where

- $\mathbf{e} \in \mathbb{R}^{|\mathcal{F}|}$ is the vector of the energy status of flexible loads, - $\mathbf{y} \in\{0,1\}^{|\mathcal{F}|}$ is the vector of availability indicators of flexible loads,

- $\mathbf{p} \in \mathbb{R}^{|\mathcal{B}|}$ is the active power injection vector,

- $\mathbf{q} \in \mathbb{R}^{|\mathcal{B}|}$ is the reactive power injection vector,

- $\mathbf{V} \in \mathbb{C}^{|\mathcal{B}|}$ is the bus voltage vector $\left(V_{b}=v_{b} e^{j \phi_{b}}\right)$.

The slack bus is fixed at the high voltage level side of the transformer connecting the distribution network to the transmission network. By convention we refer to this bus as bus 0 , and impose $v_{0}=1$ p.u. and $\phi_{0}=0$.

\section{Feasible states}

To be feasible the state $x_{n}$ must reside within the set $\mathcal{X}_{n}$ defined by:

- $\forall b \in \mathcal{B}$, the active and reactive power definition

$$
p_{b, n}+j q_{b, n}=V_{b, n} \sum_{b^{\prime} \in \mathcal{B}} Y_{b, b^{\prime}}^{*} V_{b^{\prime}, n}^{*}
$$

- $\forall b \in \mathcal{B}$, the voltage limits

$$
\underline{V}_{b} \leq v_{b, n} \leq \bar{V}_{b}
$$

- $\forall l \in \mathcal{L}$, the current limits ( $l$ connects buses $b$ and $b^{\prime}$ )

$$
\left|Y_{b b^{\prime}}\left(V_{b, n}-V_{b^{\prime}, n}\right)\right| \leq \bar{I}_{l},
$$

- if $n$ is a leaf of the tree, the energy constraint of flexible orders

$$
e_{f, n}=\sum_{k \in \text { scenario }(n)} P_{f, k} \Delta t, \forall f \in \mathcal{F}
$$

\section{Control actions}

The first stage decision $u_{0}$ encompasses the flexibility procurement decisions, $u_{f, 0}, \forall f \in \mathcal{F}$. For stages 1 to $T$ the control variable is $u=(\mathbf{c}, \mathbf{m})$, where $\mathbf{c} \in \mathbb{R}^{|\mathcal{G}|}$ is the vector of curtailment instructions for the generation units and $\mathbf{m} \in \mathbb{R}^{|\mathcal{F}|}$ is the vector of flexible load power modulations.

\footnotetext{
${ }^{2}$ The operator $|\cdot|$ applied to a set returns its cardinality.
}

\section{Feasible control actions}

The control actions $u_{n^{\prime}}$ are restricted to the set $\mathcal{U}_{n^{\prime}}\left(x_{n}\right)$, where $n$ is the parent node of $n^{\prime}$. This set is defined by

- $\forall g \in \mathcal{G}$, the generator curtailment limits

$$
c_{g, n^{\prime}} \in[0,1]
$$

- $\forall f \in \mathcal{F}$, the modulation limits of the flexible loads

$$
m_{f, n^{\prime}} \in\left[\frac{\underline{P}_{f, n^{\prime}}-P_{f, n^{\prime}}}{P_{f, n^{\prime}}} y_{f, n}, \frac{\bar{P}_{f, n^{\prime}}-P_{f, n^{\prime}}}{P_{f, n^{\prime}}} y_{f, n}\right] \text {. }
$$

For the first stage control action $u_{0}$, the flexibility provision variables are binary: $u_{0, f} \in\{0,1\}, \forall f \in \mathcal{F}$.

\section{Transition function}

The transitions $x_{n^{\prime}}=f\left(x_{n}, u_{n^{\prime}}\right)$ are defined by, $\forall n^{\prime} \in \mathcal{C}(n)$ and $\forall n \in \mathcal{N} \backslash\{0\}$,

- the evolution of the energy provided to flexible loads

$$
e_{f, n^{\prime}}=e_{f, n}+\left(1+m_{f, n^{\prime}}\right) P_{f, n^{\prime}} \Delta t, \forall f \in \mathcal{F}
$$

where $\Delta t$ is the period duration

- the propagation of the load flexibility availability indicator

$$
y_{f, n^{\prime}}=y_{f, n}, \forall f \in \mathcal{F}
$$

- the net active and reactive power injections, $\forall b \in \mathcal{B}$

$$
\begin{aligned}
p_{b, n^{\prime}} & =\sum_{g \in \mathcal{G}(b)} c_{g, n^{\prime}} P_{g, n^{\prime}} \\
& -\sum_{s \in \mathcal{S}(b)} P_{s, n^{\prime}}-\sum_{f \in \mathcal{F}(b)}\left(1+m_{f, n^{\prime}}\right) P_{f, n^{\prime}} \\
q_{b, n^{\prime}} & =\sum_{g \in \mathcal{G}(b)} c_{g, n^{\prime}} Q_{g, n^{\prime}} \\
& -\sum_{s \in \mathcal{S}(b)} Q_{s, n^{\prime}}-\sum_{f \in \mathcal{F}(b)}\left(1+m_{f, n^{\prime}}\right) Q_{f, n^{\prime}}
\end{aligned}
$$

Finally, $\forall n \in \mathcal{C}(0)$, we have simply $y_{f, n}=u_{f, 0}$.

\section{Objective function}

The first term of the objective function corresponds to the cost of procuring the availability of flexible loads in advance. The second term expresses the cost of the forthcoming energy curtailment decisions.

$$
J(\mathbf{x}, \mathbf{u})=\sum_{f \in \mathcal{F}} y_{f} C_{f}^{\text {flex }}+\sum_{n \in \mathcal{N}} w_{n} \sum_{g \in \mathcal{G}}\left(1-c_{g, n}\right) P_{g, n} C_{g, t}^{\text {curt }} .
$$

\section{Nature of the optimization problem}

The presence of flexibility procurement decisions and $\mathrm{AC}$ power flow equations result in a mixed integer and non-linear program (MINLP). Furthermore, the dependency between periods and the explicit modeling of uncertainty dramatically 
increase the dimensions of the problem. In the following sections, we describe results obtained on a small test system, with a short time horizon and a moderate number of scenarios. In the concluding section, we discuss pitfalls and avenues for solving realistic scale instances.

\section{Case Study}

We analyze issues arising at the MV level in some Belgian distribution systems (Figure 5). Often, wind-farms are directly connected to the HV/MV transformer, as modeled in our test system by the generator connected to bus 2. Power off-takes and injections induced by residential consumers are aggregated at bus 4 by a load and a generator representing the total production of PV panels. Finally, the load connected to bus 5 represents an industrial consumer.

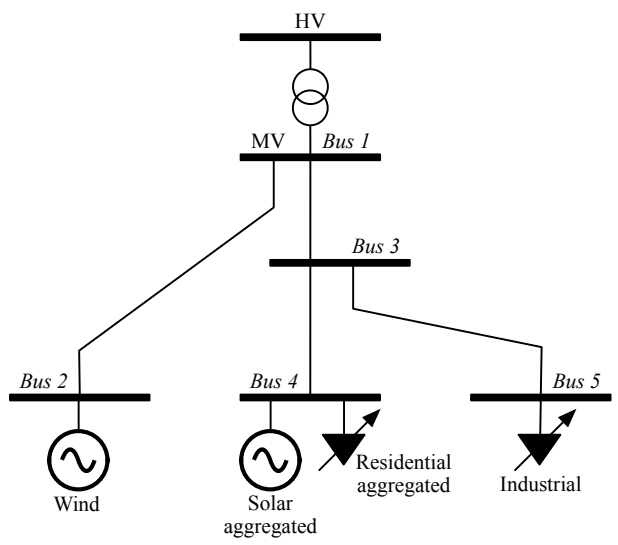

Fig. 5: Model of distribution network used for the case study.

The cumulative capacity of DG units exceeds the capacity of the HV/MV transformer. This leads to congestion issues when, within the distribution network, high generation and low consumption arise simultaneously. Voltage rises can also be induced in the downstream nodes because the power flow is mainly directed towards the transformer. On the other hand, when the local generation level is low and loads consumption is high, the power flow is inverted, and this can lead to undervoltage problems.

The optimization horizon covers three periods. The procurement of load flexibility occurs before the first period. The stochastic process associated with this time horizon relates to the uncertainty on the production of renewable generators. It is modeled through two random variables $\mathcal{W}$ and $\mathcal{S}$ which define the efficiency factors of wind and PV generators, respectively (cf. Figure 6a). The actual output level of a DG unit is thus its maximum capacity scaled by the associated efficiency factor. Figure 6a shows the scenario tree used for this case study, comprising eight possible scenarios. As both random variables model natural phenomena (wind level and sunshine), we expect uncertainty to increase as we move away from real-time. This is modeled by an increase in the standard deviation associated with the random process, as shown on

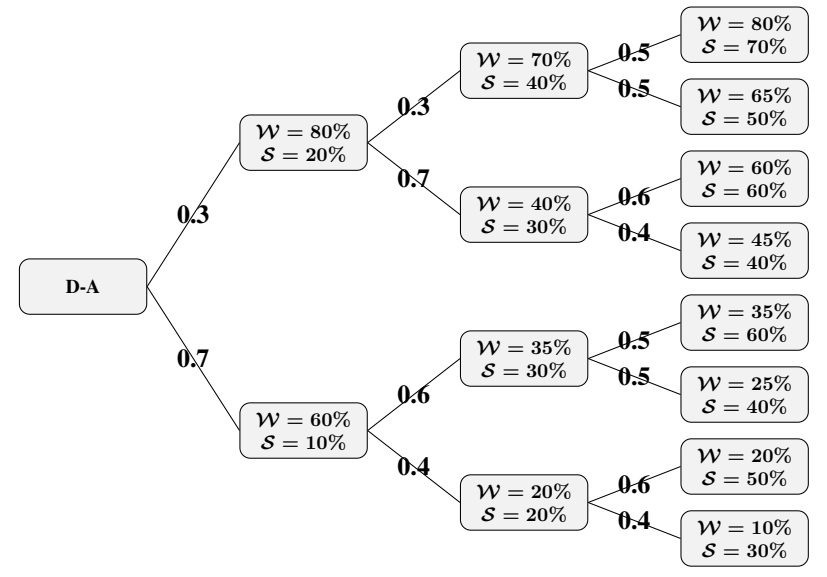

(a) Scenario tree used for the case study. The nodes show the values of the random variables and the label on the edges define the transition probabilities between nodes.

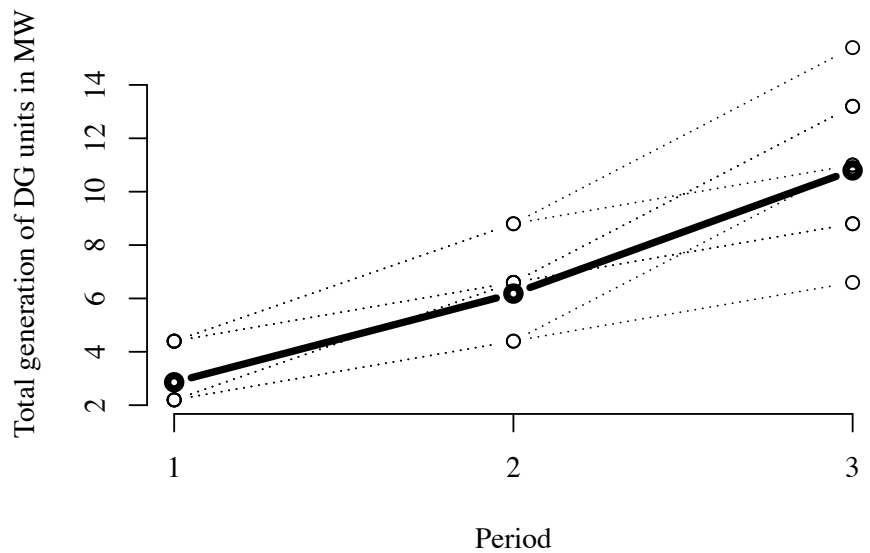

(b) Cumulative level of power injected by the DG vs. time. Dotted lines represent scenarios and the solid line corresponds to the mean scenario.

Fig. 6: Representation of uncertainty.

Figure 6b. Unlike renewable generators, the two loads have peak consumption during the first two periods. However, both can provide flexibility. The baseline demand profile and the upward and downward modulation limits are shown in Figure 7 . We define the flexibility price, $p_{f}$, such that the flexibility fees at buses 4 (aggregated residential load) and 5 (industrial load) equal $p_{f}$ and $1.5 p_{f}$, respectively.

We compare two sequential decision-making policies:

- the mean scenario approach (MSA): the procurement of flexibility is first determined by optimizing over the mean scenario. The mean scenario is updated at each recourse stage, and we solve an optimization problem for each stage while following the nodes defining the scenario in the tree and fixing the variables related to former periods (i.e., ancestor nodes). This method is evaluated for each scenario of the case study.

- the scenario tree approach (STA): this policy solves the problem by optimizing over the whole scenario tree, as described in formulation (15)-(20). Load flexibility is also evaluated using this stochastic formulation. 


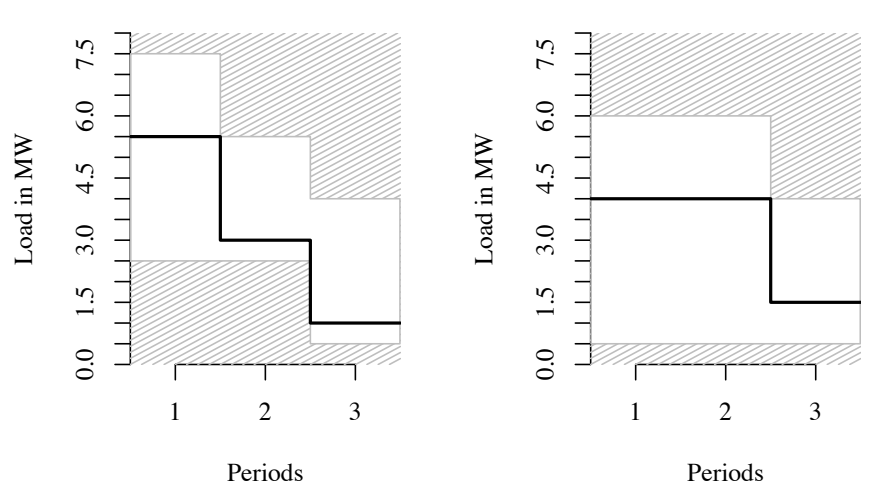

Fig. 7: Flexible loads. The white area is delimited by $\underline{P}$ and $\bar{P}$, and the black line is the baseline consumption $P$.

\section{Experimental results}

We first illustrate for one scenario the control actions taken by both approaches. The approaches are then compared in terms of expected cost and variability of cost. Finally, we show the sensitivity of the first stage and of curtailment decisions to the price of flexibility.

\section{Scenario analysis}

The selected scenario is presented in Figure 8. Without any

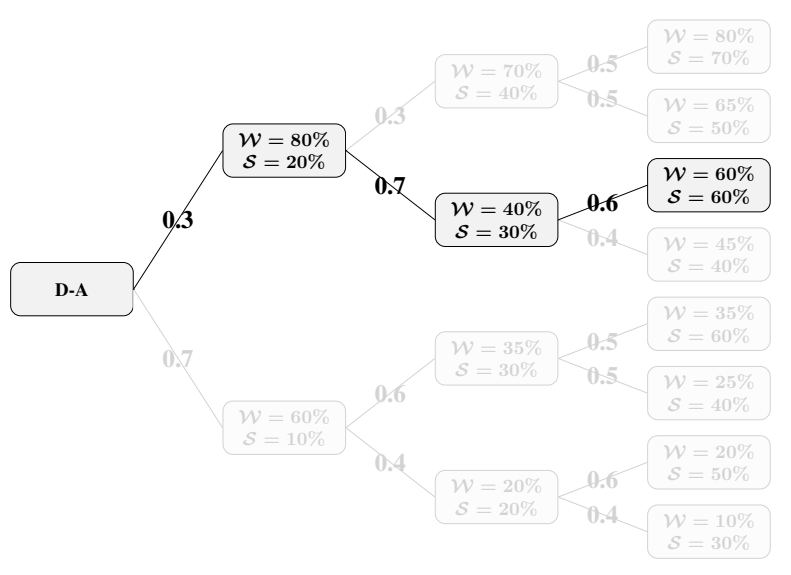

Fig. 8: Selected scenario.

action from the DSO, two operational limits would be violated during the third period (Figure 9):

- the magnitude of the current in the transformer would exceed the 10 p.u. limit (11.53 p.u.);

- an over-voltage would appear at bus 4 (1.054 p.u. for a maximum of 1.05 p.u.).

The MSA does not procure flexibility while the STA only procures the flexibility of the residential load. Thus, for the MSA, maintaining the system within the operational margins can only be ensured through curtailment orders: power from bus 2 is curtailed, at a cost of $\$ 224$. On the other hand, as

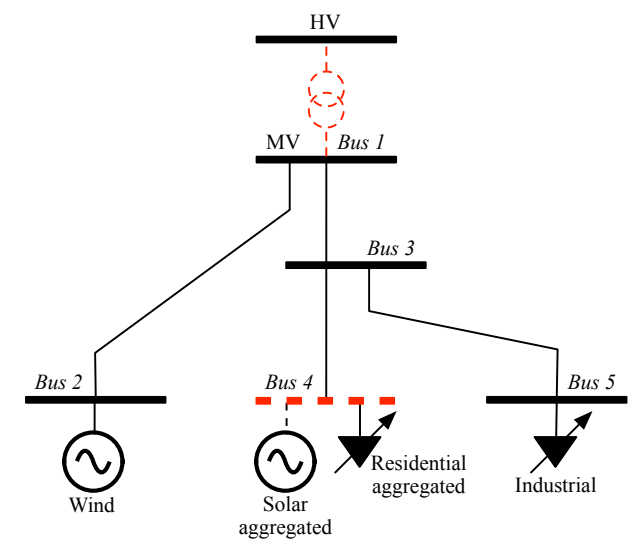

Fig. 9: Operational limit violations are located at the reddashed elements.

the STA procures flexibility from the load at bus 4 at a cost of $\$ 30$, curtailment is not required to keep the system within acceptable bounds for this scenario. The consumption of the load instructed by the DSO is presented in Figure 10.

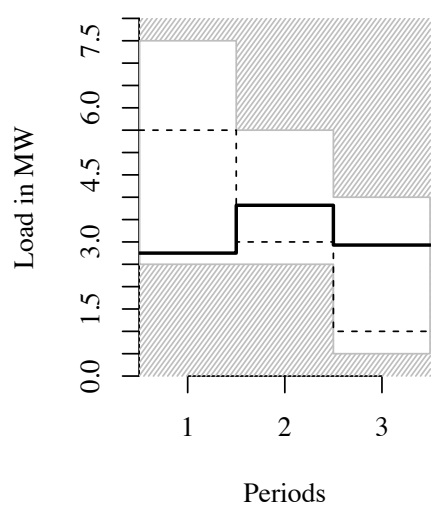

Fig. 10: Consumption pattern instructed by the DSO (line), and baseline consumption (dashed line).

\section{Features of the stochastic model}

The expected cost of operation achieved over all the scenarios for both the MSA and the STA, as well as the maximum and minimum costs among possible scenarios, are presented in Table I. The smallest expected cost of operation was achieved by the STA. In addition, the STA also ensures a smaller variability of the cost over the set of scenarios. On the contrary, as the MSA overfits its decisions to the mean scenario, it achieves zero cost on scenarios close to the mean scenario, but this is at the expense of large curtailment costs on several possible scenarios far from the mean.

\begin{tabular}{|c|c|c|c|c|}
\hline & $\mathbb{E}\{$ cost $\}$ & max cost & min cost & std. dev. \\
\hline MSA & $73 \$$ & $770 \$$ & $\mathbf{0} \$$ & $174 \$$ \\
STA & $\mathbf{4 6} \$$ & $\mathbf{3 7 9} \$$ & $30 \$$ & $\mathbf{7 2 \$}$ \\
\hline
\end{tabular}

TABLE I: Results for the MSA and the STA over all the scenarios (best value in bold, column-wise). 


\section{Features of load flexibility}

We now analyze the impact of load flexibility in terms of curtailed power from DG units and cost of operation. Figure 11 shows the evolution of the expected percentage of curtailed power, the number of activated flexible loads and the expected cost of operation, when the flexibility price $p_{f}$ increases and the curtailment prices remain constant. We observe that a very low flexibility price induces the activation of both loads, and the DSO can avoid issuing curtailment orders. At a given threshold, the flexibility fee of the industrial load (bus 5) becomes too high and only one load is activated. With only one flexible load, the DSO may have to curtail power, depending on the scenario, which thus increases the expected cost. When the price of flexibility becomes too high, the only action taken by the DSO is generation curtailment.

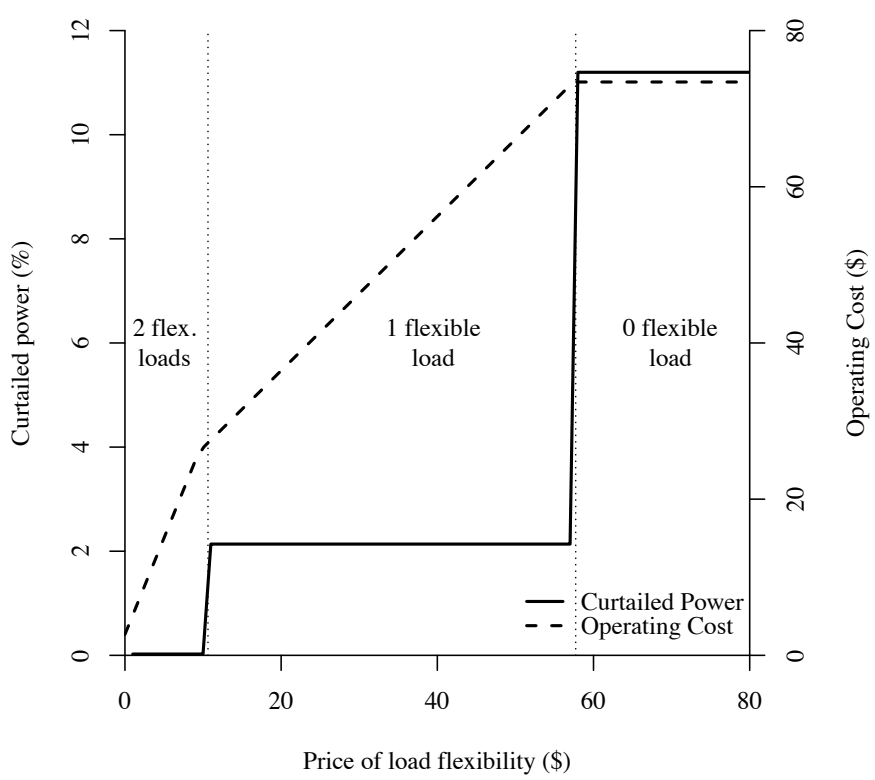

Fig. 11: Load flexibility cost analysis.

Hence, if the load flexibility price and curtailment prices are competitive, a DSO can reduce both the cost of operation and the amount of power curtailed from the distributed generators.

\section{Implementation and algorithmic details}

It is not easy to find off-the-shelf solvers that can manage this MINLP, even on such a small test system. We conducted several experiments with SCIP [13] (with and without IPOPT [14] to solve node relaxations) and Knitro [15]. Finally, we decided to implement a custom branch-and-bound algorithm that can use both IPOPT and Knitro to solve the NLP node relaxations. We observed that solutions of the optimization programs were insensitive to the choice of the NLP solver.

\section{Conclusions and future work}

This paper proposes a novel formulation of the ANM problem that allows utilization of the demand-side operational flexibility. We highlighted both the sequential and the uncertain nature of this ANM problem and addressed it as an optimal sequential decision-making problem under uncertainty. As the scope of this paper is to serve as a proof-of-concept, we benchmarked the proposed approach on a small case study, and showed that it is critical to explicitly take into account the uncertainty to efficiently operate demand-side flexibility.

The model governing the interactions between the stakeholders of the distribution system must be carefully considered. We have presented one particular flexibility model, where availability is procured one day ahead in exchange for an availability fee, and curtailed power is remunerated at the market price. In the next steps of this work, it would be worth analyzing alternative models to identify the ones that offer incentives to foster demand-side flexibility and increase the share of renewable energy. The flexibility product that we proposed may fit a subset of the loads, but other products may be required to satisfy the technical constraints of other consumers. For example, this work could be easily extended to tank-like models, such as the one proposed in [16]. Furthermore, existing configurations could already enable activation of the demand flexibility through ON/OFF signals that actuate a relay in some domestic meters (such as day and night meters). However, these models may necessitate more specific information on the consumption side, and introduce more integer variables to the optimization problem.

More or less independently of the interaction model implemented, computational challenges are a barrier to the application of this formulation to real size systems. Indeed, the complexity of the optimization problem is simultaneously increased by the integer variables, the non-linearity of the power flow equations, and the high dimensionality caused jointly by the multi-period aspect and the explicit treatment of uncertainty. It is certainly worth considering the recent works that propose new approaches for efficiently solving single period OPF problems [17], [18]. Another interesting research direction would be to develop optimization algorithms specifically designed to tackle the complexity induced by the scenario-tree based approach [19], [20].

In the long-term, it will also be necessary to find investment strategies that reach the best possible trade-off between the fitand-forget and full ANM approaches. Assessing such a tradeoff may not be an easy task, especially since it would require the evaluation of the cost of an ANM scheme, which may be a difficult task. Note that this cost depends not only on the price paid for modulating the load and the generation over a long period of time, but also on the cost required for the evolution of the infrastructure. Indeed, deploying these types of approaches would require non-negligible ICT, metering and control investments, amongst others. 


\section{Acknowledgment}

This research is supported by the Public Service of Wallonia - Department of Energy and Sustainable Building within the framework of the GREDOR project.

These authors also acknowledge the financial support of the Belgian Network DYSCO, an Interuniversity Attraction Poles Programme initiated by the Belgian State, Science Policy Office.

\section{References}

[1] D. Fouquet and T. B. Johansson, "European renewable energy policy at crossroads - Focus on electricity support mechanisms," Energy Policy, vol. 36, no. 11, pp. 4079-4092, 2008.

[2] J. A. P. Lopes, N. Hatziargyriou, J. Mutale, P. Djapic, and N. Jenkins, "Integrating distributed generation into electric power systems: A review of drivers, challenges and opportunities," Electric Power Systems Research, vol. 77, no. 9, pp. 1189-1203, 2007.

[3] A. Keane, Q. Zhou, J. W. Bialek, and M. O'Malley, "Planning and operating non-firm distributed generation," IET Renewable Power Generation, vol. 3, no. 4, pp. 455-464, 2009.

[4] F. Capitanescu, J. L. Martinez Ramos, P. Panciatici, D. Kirschen, A. Marano Marcolini, L. Platbrood, and L. Wehenkel, "State-of-theart, challenges, and future trends in security constrained optimal power flow," Electric Power Systems Research, vol. 81, no. 8, pp. 1731-1741, 2011.

[5] S. N. Liew and G. Strbac, "Maximising penetration of wind generation in existing distribution networks," IEE Proceedings: Generation, Transmission and Distribution, vol. 149, no. 3, pp. 256-262, 2002.

[6] M. J. Dolan, E. M. Davidson, I. Kockar, G. W. Ault, and S. D. J. McArthur, "Distribution power flow management utilizing an online optimal power flow technique," IEEE Transactions on Power Systems, vol. 27, no. 2, pp. 790-799, 2012.
[7] L. F. Ochoa, C. J. Dent, and G. P. Harrison, "Distribution network capacity assessment: Variable DG and active networks," IEEE Transactions on Power Systems, vol. 25, no. 1, pp. 87-95, 2010.

[8] L. F. Ochoa and G. P. Harrison, "Minimizing energy losses: Optimal accommodation and smart operation of renewable distributed generation,' IEEE Transactions on Power Systems, vol. 26, no. 1, pp. 198-205, 2011

[9] L. F. Ochoa and G. P. Harrison, "Using AC optimal power flow for DG planning and optimisation," in IEEE PES General Meeting, 2010.

[10] H. Klinge Jacobsen and S. T. Schröder, "Curtailment of renewable generation: Economic optimality and incentives," Energy Policy, vol. 49, no. C, pp. 663-675, 2012.

[11] S. W. Wallace and S.-E. Fleten, "Stochastic programming models in energy," Handbooks in operations research and management science, vol. 10, pp. 637-677, 2003.

[12] A. Shapiro, D. Dentcheva, and A. Ruszczyński, Lectures on stochastic programming: modeling and theory. SIAM, 2009.

[13] T. Achterberg, "SCIP: solving constraint integer programs," Mathematical Programming Computation, vol. 1, pp. 1-41, Jan. 2009.

[14] A. Wächter and L. Biegler, "On the implementation of a primaldual interior point filter line search algorithm for large-scale nonlinear programming," Mathematical Programming, vol. 106, no. 1, pp. 25$57,2006$.

[15] R. Byrd, J. Nocedal, and R. Waltz, "Knitro: an integrated package for nonlinear optimization," Large-scale nonlinear optimization, pp. 1-25, 2006.

[16] S. Mathieu, D. Ernst, and Q. Louveaux, "An efficient algorithm for the provision of a day-ahead modulation service by a load aggregator." Preprint, 2013.

[17] J. Lavaei and S. H. Low, "Zero Duality Gap in Optimal Power Flow Problem," IEEE Transactions on Power Systems, vol. 27, pp. 92-107, Feb. 2012.

[18] D. Phan, "Lagrangian duality and branch-and-bound algorithms for optimal power flow," Operations Research, vol. 60, pp. 275-285, May 2012.

[19] B. Cornélusse, G. Vignal, B. Defourny, and L. Wehenkel, "Supervised learning of intra-daily recourse strategies for generation management under uncertainties," in PowerTech, 2009 IEEE Bucharest, pp. 1-8, IEEE, 2009.

[20] B. Defourny, D. Ernst, and L. Wehenkel, "Multistage stochastic programming: A scenario tree based approach to planning under uncertainty," Decision theory models for applications in artificial intelligence: concepts and solutions, Sucar LE, Morales EF, Hoey J (eds). Information Science Publishing: Hershey, Pennsylvania, USA, 2011. 